Casos Clínicos

Arch. Esp. Urol., 61, 7 (825-827), 2008

\title{
TUMOR TESTICULAR DE LOS CORDONES SEXUALES NO ESPECÍFICO. PRESENTACIÓN DE UN CASO
}

Oscar Miranda Aranzubía, Jorge García Rodríguez, Roberto Carlos González, Francisco Javier Martínez Gómez, Jesús María Fernandez Gómez y Francisco Javier Regadera Sejas.

Servicio de Urología. Hospital Universitario Central de Asturias. Oviedo. Asturias. España.

Resumen.- OBJETIVOS: Presentamos el caso de un tumor testicular del estroma gonadal y revisamos la literatura al respecto.

MÉTODOS/RESULTADOS: Presentamos el caso de un varón de 42 años que consulta por bultoma indoloro en testiculo derecho, practicándose orquiectomía y seguimiento.

CONCLUSIONES: Los tumores de los cordones sexuales no específicos son una entidad extremadamente rara, con un crecimiento lento y comportamiento benigno, que presentan psitvidad para distintos marcadores.

Palabras clave: Tumor testicular. Estroma gonadal.

Oscar Miranda Aranzubía

Servicio de Urología

Hospital Universitario Central de Asturias

Oviedo Asturias. (España).

mirandaaranzubia@gmail.com

坾

Trabajo recibido: 26 de noviembre 2007. 
Summary.- OBJECTIVE: We report one case of gonadal stromal testicular tumor and perform a bibliographic review.

METHODS/RESULTS: We present the case of a 42-year-old male patient consulting for a painless right testicular mass. Orchiectomy was performed and the patient underwent follow-up.

CONCLUSIONS: Nonspecific sexual cord tumors are extremely rare, with slow growing and benign behaviour, presenting positive staining for various markers.

Keywords: Testicular tumor. Gonadal stroma.

\section{INTRODUCCIÓN}

El tumor de testículo es una patología poco frecuente en nuestro medio, con una incidencia estimada en Estados Unidos de 2-3 casos por 100.000 habitantes y año (1). En España se encuentra situado sólo por delante de algunos tumores muy poco frecuentes como el tumor de vesícula biliar o el mieloma (2). De entre los distintos tipos histológicos, los tumores del estroma gonadal son excepcionales, ya que representan únicamente un $3 \%$ de los tumores testiculares.

En la literatura existen únicamente 11 casos que se pueden encuadrar dentro de la definición de tumor de los cordones sexuales no específico de celulas de Sertoly o de Leydig. Presentamos el caso de un paciente adulto que consulta por hallazgo casual de nódulo testicular cuyo diagnóstico anatomopatológico es compatible con tumor fusiforme del estroma sexual no específico.

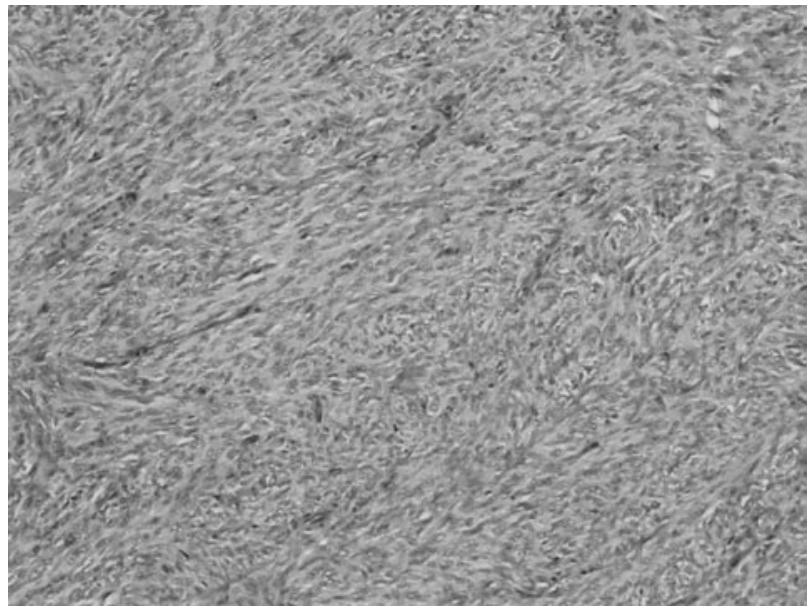

FIGURA 1. Tumor de células fusiformes (HE).

\section{CASO CLÍNICO}

Se trata de un varón de 42 años sin antecedentes médicos de interés, y con una cirugía de vasectomía como único antecedente urológico. Acudió a nuestra consulta por hallazgo casual de un nódulo en testículo derecho.

Ante dicha exploración se solicita ecografía que nos informa de una imagen bien delimitada, de densidad homogénea, hipoecoica, localizada en polo superior de teste derecho. Los marcadores tumorales alfa-feto proteína, beta-HCG, resultaron dentro de la normalidad, así como el resto de la exploración física, donde no se objetivó signos de sobreproducción hormonal.

Se decide por tanto exploración quirúrgica del mismo, realizándose biopsia intraoperatoria de dicho nódulo, que se informa como tumor fusiforme de bajo grado sin poder descartar malignidad, por lo que se decide practicar orquiectomía radical derecha vía inguinal.

El paciente presentó una buena evolución postoperatoria siendo dado de alta al día siguiente. El informe definitivo anatomopatológico fue de tumor fusiforme del estroma sexual no específico.

En el seguimiento posterior de este paciente no se ha objetivado signos de recurrencia o progresión.

\section{DISCUSIÓN}

Los tumores del estroma gonadal no específicos (TEG$\mathrm{NE}$ ) constituyen una entidad poco frecuente dentro de la patología tumoral testicular.

Estos engloban una serie de lesiones compuestas por una desigual proporción de tumor testicular de células

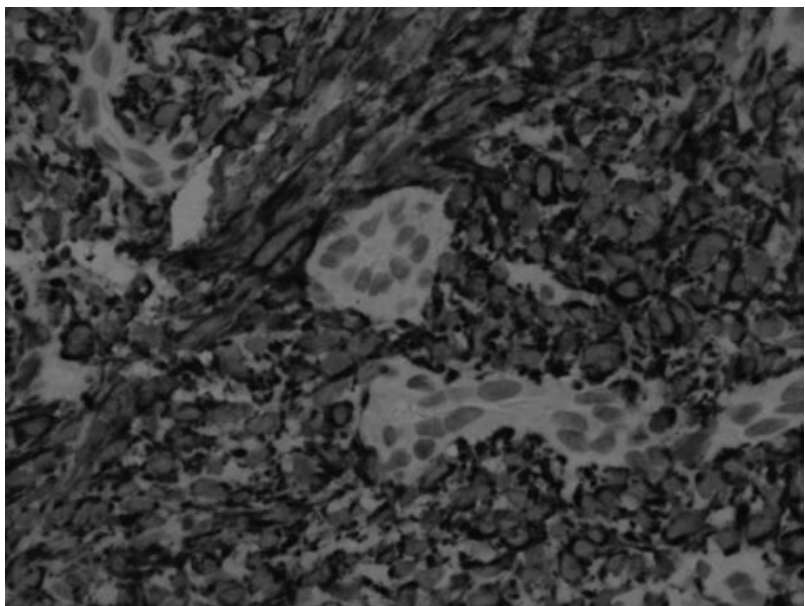

FIGURA 2. Citoqueratinas de amplio espectro. 


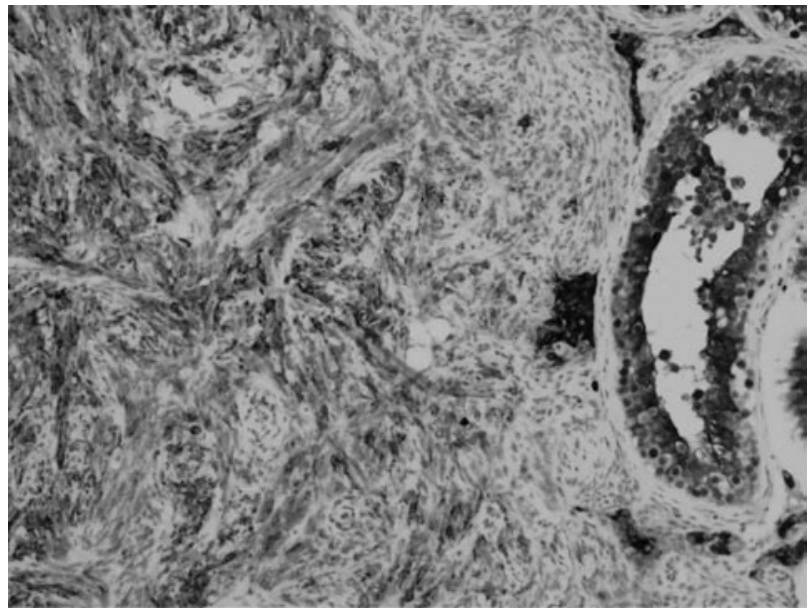

FIGURA 3. Inmunohistoquímica de Inhibina.

de los cordones sexuales, sin un fenotipo claro de células de Leydig o de Sertoli, por lo que se ha dado también en llamar a esta entidad como fibroma testicular, en analogía con el fibroma ovárico. Se trata de tumores con unas características definidas que quedan recogidas en la Tabla 1.

Son tumores pequeños, redondeados con células fusiformes y colágeno (Figura 1). De consistencia firme y blanquecina, con positividad para distintos marcadores como son MIC2, S100, Vimentina, Inhibina, Desmina y citoqueratinas (Figura 2) y con escasa actividad mitótica (1-4 mitosis por campo de gran aumento) (4).

Este tipo de lesión tiene generalmente un comportamiento benigno e indolente, con un crecimiento lento, siendo el síntoma más frecuente de consulta el hallazgo casual de un nódulo testicular.

TABLA I. TUMORES DEL ESTROMA TESTICULAR NO ESPECÍFICOS.

- $39+$ +/- 4.2 años (rango 16-67)

- Sin síntomas hormonales

- Sin rasgos clínicos característicos

- Marcadores (aFP, beta-HCG) normales

- Detectados por palpación

- Entre 0,4 y $5 \mathrm{~cm}$.

- En el parénquima sin afectar la albugínea.
El diagnóstico se obtiene mediante histología y técnicas de inmunohistoquímia (Figura 3), lo que nos ayuda a realizar el diagnóstico diferencial con lesiones malignas de otra estirpe.

A pesar de su comportamiento benigno precisan un seguimiento a largo plazo, mediante la tac y marcadores tumorales. Ya que, aunque en la bibliografía revisada no se ha descrito casos de malignización en este tipo de tumores, la poca casuística y la posibilidad de que se trate de otra estirpe maligna, exigen una vigilancia (5).

\section{CONCLUSIÓN}

Los tumores de los cordones sexuales no específicos son una entidad extremadamente rara, con un crecimiento lento y comportamiento benigno, que presentan psitvidad para distintos marcadores.

\section{BIBLIOGRAFIA y LECTURAS \\ RECOMENDADAS (*lectura de interés $y^{* *}$ lectura fundamental)}

1. SANTOS ARRONTES, D.; PAEZ BORDÁ, A. y cols.: "Incidencia del cancer urológico en un área sanitaria de 300000 habitantes". Actas Urol. Esp., 28: 646, 2004.

2. LOPEZ-ABENTE, G.; POLLÁN, M. y cols.: "Situación del cancer en España: Incidencia”. An. Sist. Sanit. Navar., 2: 27, 2004.

3. DE PINEUX, G.; GLASER, C. y cols.: "Testicular fibroma of gonadal stromal origin with minor sex cord elements". Arch. Pathol. Lab. Med., 123, 1999.

4. TARJEN, M.; SARKISSOV, G.; TOT, T.: "Unclasified sex cord/gonadal stromal testis tumor with predominance of spindle cels". APMIS, 114: 465, 2006.

5. JIMENEZ CRUZ, J.F.; RIOJA SANZ, L.A.: "Tratado de urología". 4: 2093. 